\title{
APPENDIX 2: VARIANT READINGS
}

\section{Based on Collations by Morgan Reed and Adam Kane}

The following tables list variant readings of the Mosul edition of the Peshitta Psalter as compared to the Leiden edition, along with a summary of manuscript support for the Mosul reading. The fourth column indicates the support for the Mosul reading as follows:
early
first millennium up to and including the tenth century.
late post tenth century making use of the Leiden ${ }^{1}$ apparatus. $^{2}$
Lee Lee's edition (1823) agrees with Mosul.
Urmia The Urmia edition (1852) agrees with Mosul.
no support Mosul's reading is unique.

\begin{tabular}{|c|c|c|c|}
\hline $\begin{array}{l}\text { Place } \\
1: 3\end{array}$ & $\begin{array}{l}\text { Mosul } \\
\text { \o }\end{array}$ & $\begin{array}{l}\text { Leiden } \\
\text { om waw }\end{array}$ & $\begin{array}{l}\text { Ms support for } \mathbf{M} \\
\text { early }\end{array}$ \\
\hline $1: 5$ & لlo & om waw & early \\
\hline $2: 3$ & مسمبا & مسع:| & late \\
\hline $2: 12$ & سمعه ح:| & أمه, مح:مال, & early \\
\hline $2: 12$ & i & הنه مهنما & early \\
\hline 3:2 [L 3:3] & مهـتتا|| & om waw & late \\
\hline 3:2 [L 3:3] & خل & pr dälath & early \\
\hline 3:3 [L 3:4] & Nio & om waw & early \\
\hline $4: 1$ [L 4:2] & محاهخيت & om waw initial & early \\
\hline 5:4 [L 5:5] & $\mathrm{Ng}$ & No & early \\
\hline $5: 10$ [L 5:11] & مسد & لسمح & early \\
\hline
\end{tabular}

${ }^{1}$ Variants in 7a1 cited in (or first in) Leiden's First Apparatus which fail to support Mosul are ignored.

2 Since the Leiden Edition does not print a diacritical dot on a $3^{\text {rd }}$ fem. sing. suffix (no matter what 7a1 has), such diacritical marks are ignored in this table. In cases of the addition or omission of a seyame Leiden has not published the evidence its collators gathered. Variant forms of verbs are commonly treated as orthographical by Leiden and not published. 
Place

5:11 [L 5:12]

5:11 [L 5:12]

6:1 [L 6:2]

6:8 [L 6:9]

6:8 [L 6:9]

7:7 [L 7:8]

7:8 [L 7:9]

7:9 [L 7:10]

7:11 [L 7:12]

7:14 [L 7:15]

8:6 [L 8:7]

8:6 [L 8:7]

8:8 [L 8:9]

9:10 [L 9:11]

9:14 [L 9:15]

10:5

10:7

10:15

10:15

10:15

10:17

11:5

11:6

12:6 [L 12:7]

12:8 [L 12:9]

$16: 2$

16:3

16:5

16:6

16:7

16:9

16:10

17:6

18:2 [L 18:3]

18:2 [L 18:3]

18:4 [L 18:5]

18:5 [L 18:6]
Mosul

حلهم

,

لlo

000:0

مس

yogal

a

حروما

No

IA

bo

Lent

حمحلا

تمد:0

حهزي

متح

Lout

موحمل

لماحده

مهم

محن|

محةقلا

oزما

مامد:

פיمب

in

9io

oa

9io

9io

9io

وبسرا

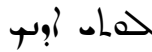

حט

م0دوب

0

متحلا
Leiden

pr waw

pr waw

om waw

00:0

م

Tal

uno,

seyāme

om waw

Var

om waw

Lut

seyāme

pr waw

مئس

pr waw

مامبـ

موحنما

ماحما

wolal

pr waw

om initial wan

om initial waw

مامد: مال

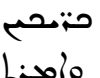

om waw

om

om waw

om waw

om waw

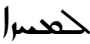

tr

pr waw

om waw

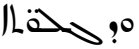

om waw
Ms support for $\mathbf{M}$

late

early

late

early

early

early

early

Urmia

no support

early

early

no support

early

early

early

early

Urmia

early

early

early

early

early

early

late

late

early

late

early

early

early

early

early

early

early

early

early

early 


\begin{tabular}{|c|c|c|c|}
\hline $\begin{array}{l}\text { Place } \\
\text { 18:6 [L 18:7] }\end{array}$ & $\begin{array}{l}\text { Mosul } \\
\text { Nح }\end{array}$ & $\begin{array}{l}\text { Leiden } \\
\text { pr waw }\end{array}$ & $\begin{array}{l}\text { Ms support for } \mathbf{M} \\
\text { early }\end{array}$ \\
\hline 18:7 [L 18:8] & $u_{1}$ & تحس & no support \\
\hline 18:7 [L 18:8] & حLlio & Llio & late \\
\hline 18:8 [L 18:9] & مخزهلا & seyāme & early \\
\hline 18:9 [L 18:10] & Lank & Lامس & Urmia \\
\hline 18:10 [L 18:11] & منقر & متوصنه & early \\
\hline 18:14 [L 18:15] & محتمع00. & om initial waw & early \\
\hline 18:15 [L 18:16] & aمانقا & dîna & early \\
\hline 18:18 [L 18:19] & ممبمهس & om initial waw & early \\
\hline 18:20 [L 18:21] & ف:حس & pr waw & early \\
\hline 18:24 [L 18:25] & ف:حس & pr waw & early \\
\hline 18:33 [L 18:34] & ;مار & ;مس & early \\
\hline 18:38 [L 18:39] & Loul & Lut & Urmia \\
\hline 18:43 [L 18:44] & محما & om waw & early \\
\hline 19:2 [L 19:3] & סגרו & om waw & early \\
\hline 19:4 & Wh & Who & early \\
\hline 19:6 [L19:7] & مـ & pr waw & early \\
\hline 19:12 [L 19:13] & حمتحد| & om bèth & early \\
\hline 19:14 [L 19:15] & ; & pr waw & early \\
\hline 20:1 [L 20:2] & asa & ممال & early \\
\hline 21:5 [L 21:6] & معحسا & acحua & late \\
\hline 21:11 [L 21:12] & iامسحه & pr waw & early \\
\hline 22:1 [L 22:2] & مس & م & early \\
\hline 22:9 [L 22:10] & مج2 & גו" & early \\
\hline 22:13 [L 22:14] & ond & om initial waw & early \\
\hline 22:14 [L 22:15] & حصs & $\vec{b}$ & early \\
\hline $24: 7$ & Werg, & om dälath & early \\
\hline $25: 2$ & $2 \mathrm{~J}$ & pr dälath & early \\
\hline $25: 3$ & 9io & om waw & early \\
\hline $25: 3$ & حم:مع|0atp & seyāme & early \\
\hline $25: 13$ & onaro & om waw & early \\
\hline $25: 18$ & مسرد & pr waw & early \\
\hline $26: 6$ & أحمى & pr waw & early \\
\hline $26: 10$ & وحامب0aم & وحلَمب00م & early \\
\hline $26: 12$ & ;هد & pr waw & early \\
\hline $27: 6$ & أوحس & pr waw & early \\
\hline 28:8 & وهمزمسا & مو:001 & early \\
\hline
\end{tabular}




\begin{tabular}{|c|c|c|c|}
\hline $\begin{array}{l}\text { Place } \\
28: 9\end{array}$ & $\begin{array}{l}\text { Mosul } \\
\text { زحس }\end{array}$ & $\begin{array}{l}\text { Leiden } \\
\text { pr waw }\end{array}$ & $\begin{array}{l}\text { Ms support for } \mathbf{M} \\
\text { early }\end{array}$ \\
\hline $29: 5$ & ومسهو & ومسرو & early \\
\hline $29: 11$ & o & om waw & early \\
\hline 30:8 [L 30:9] & من:حمب & pr waw & early \\
\hline 31:1 [L 31:2] & محروسمهاب & om waw initial & early \\
\hline 31:8 [L 31:9] & SToro & om waw & early \\
\hline 31:10 [L 31:11] & a:Dlio & om waw & early \\
\hline 31:23 [L 31:24] & لمهaتا & pr waw & early \\
\hline $32: 8$ & مأوبر & om waw initial & early \\
\hline $33: 3$ & مون: & pr waw & early \\
\hline $33: 7$ & مسمه & صטص & Urmia \\
\hline $33: 7$ & حاهزן & seyāme & late \\
\hline $33: 7$ & & om seyāme & late \\
\hline $33: 9$ & 000 & 0.000 & early \\
\hline $33: 16$ & Vloio & om waw & early \\
\hline $33: 19$ & ومפ & وبوا & early \\
\hline 34:6 [L 34:7] & La & sa & early \\
\hline 34:7 [L 34:8] & مهور & مهفيا & early \\
\hline $35: 19$ & U & pr dälath & early \\
\hline $35: 20$ & حازخا & ا? & late \\
\hline $35: 22$ & U & pr waw & early \\
\hline $35: 24$ & 90, حس & وסس & early \\
\hline 36:6 [L 36:7] & aiof & $1 \ddot{0}$ & early \\
\hline 36:6 [L 36:7] & ladi? & om dälath & early \\
\hline $37: 25$ & Noio & om waw & early \\
\hline $37: 39$ & مهب:; & om waw & early \\
\hline $37: 39$ & حرحتا & om seyāme & early \\
\hline 38:1 [L 38:2] & لloio & om waw & early \\
\hline 38:3 [L 38:4] & ح & pr waw & early \\
\hline 38:5 [L 38:6] & م & pr waw & early \\
\hline 39:2 [L 39:3] & 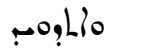 & $\operatorname{Nog}_{0}$ & early \\
\hline 39:2 [L 39:3] & أمبما & iاوبحس & early \\
\hline 39:6 [L 39:7] & مهه & pr waw & early \\
\hline 39:8 [L 39:9] & م & pr waw & early \\
\hline 39:8 [L 39:9] & مهب! & pr waw & early \\
\hline 39:10 [L 39:11] & סمس & م) & early \\
\hline 40:1 [L 40:2] & ماهس & ?ili? & early \\
\hline
\end{tabular}




\begin{tabular}{|c|c|c|c|}
\hline $\begin{array}{l}\text { Place } \\
40: 4 \text { [L 40:5] }\end{array}$ & $\begin{array}{l}\text { Mosul } \\
\text { Ulo }\end{array}$ & $\begin{array}{l}\text { Leiden } \\
\text { om waw }\end{array}$ & $\begin{array}{l}\text { Ms support for } \mathbf{M} \\
\text { early }\end{array}$ \\
\hline 40:6 [L 40:5] & مهزتحل & محمهةحل & early \\
\hline 40:13 [L 40:14] & لموفيماس & لمهو, & early \\
\hline 41:1 [L 41:2] & حسما & pr dälath & early \\
\hline 41:6 [L 41:7] & & om waw & Urmia \\
\hline 41:10 [L 41:11] & 40:910 & uo:9i, & early \\
\hline 42:1 [L 42:2] & مa & مo & Urmia \\
\hline 42:3 [L 42:4] & أحه & pr dälath & early \\
\hline 42:6 [L 42:7] & مـ2 & pr waw & early \\
\hline 42:8 [L 42:9] & $\mid L \alpha_{3}$ & or $\mid L \alpha_{3}$ & early \\
\hline 42:10 [L 42:11] & र & om waw & early \\
\hline 44:1 [L 44:2] & 910 & om waw & early \\
\hline 44:3 [L 44:4] & Noio & om waw & early \\
\hline 44:6 [L44:7] & Ulo & om waw & early \\
\hline 44:9 [L 44:10] & $\ln 00$ & om waw & early \\
\hline 44:14 [L 44:15] & مذه & pr waw & early \\
\hline $44: 26[44: 27]$ & حبין & حب: حس & early \\
\hline $44: 26[44: 27]$ & 00:00مى & مو:00مس & early \\
\hline 45:1 [L 45:2] & حمحا| & om initial làmadh & early \\
\hline 45:2 [L 45:3] & مون: & pr dälath & early \\
\hline 45:4 [L 45:5] & مهمحما & om initial waw & early \\
\hline 45:6 [L 45:7] & مهزمس الحها & مهزمسه بilal & early \\
\hline 45:9 [L 45:10] & م & pr waw & early \\
\hline 45:13 [L 45:14] & مهاه & pr waw & early \\
\hline 45:13 [L 45:14] & aina & معحسا & early \\
\hline 45:14 [L 45:15] & منهحه| & مهتخ & early \\
\hline 46:9 [L 46:10] & oمحرل" & om waw & early \\
\hline 48:8 [L 48:9] & lea & 2a & Urmia \\
\hline 48:12 [L 48:13] & dow;olit & is:0م:0م & early \\
\hline 48:13 [L 48:14] & أمن:ا & أمن:ا & early \\
\hline 48:14 [L 48:15] & oog, & 000 & early \\
\hline 48:14 [L 48:15] & لبحنן & ب:س & early \\
\hline 49:4 [L 49:5] & حصن:| & seyāme & early \\
\hline 49:9 [L 49:10] & مLLما & ومار & early \\
\hline 49:17 [L 49:18] & lloio & om waw & early \\
\hline 49:18 [L 49:19] & os, & om waw & early \\
\hline 49:19 [L 49:20] & سمره & سرا & early \\
\hline
\end{tabular}




\begin{tabular}{|c|c|c|c|}
\hline $\begin{array}{l}\text { Place } \\
\text { 49:20 [L 49:21] }\end{array}$ & $\begin{array}{l}\text { Mosul } \\
\text { حلمعنه }\end{array}$ & $\begin{array}{l}\text { Leiden } \\
\text { pr dälath }\end{array}$ & $\begin{array}{l}\text { Ms support for } \mathbf{M} \\
\text { early }\end{array}$ \\
\hline 50:9 & لما0 & om waw & early \\
\hline $50: 23$ & ماolo & om waw & early \\
\hline 51:16 [L 51:18] & لlo & pr waw & early \\
\hline 51:19 [L 51:21] & r:00 & om waw & early \\
\hline 52:5 [L 52:7] & لحمن: & لحم:000. & early \\
\hline 52:5 [L 52:7] & منمسوب & 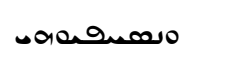 & early \\
\hline 52:5 [L 52:7] & ممصب & ممصده & early \\
\hline 52:5 [L 52:7] & محمن: & o:10:0 & early \\
\hline 53:3 [L 53:4] & لماo & om waw & early \\
\hline 54:1 [L 54:3] & 9. حص & 9wo" & early \\
\hline $55: 7$ & oو:م: & om waw & early \\
\hline 55:7 [L 55:8] & 0و:مـ & om waw & late \\
\hline 55:12 [L 55:13] & مأهلا & om waw & early \\
\hline 56:1 [L 56:2] & معار & pr waw & early \\
\hline $56: 2$ [L 56:3] & illLi & piLLi & Lee \\
\hline 56:13 [L 56:14] & متجد| & مهزي & early \\
\hline 57:8 [L 57:9] & 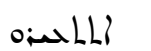 & ? & Lee, Urmia \\
\hline 58:1 [L 58:2] & ह & 3 & early \\
\hline 58:11 [L 58:12] & مهامن: & om waw & early \\
\hline 58:11 [L 58:12] & oa & صמ & early \\
\hline 58:11 [L 58:12] & Nio & om waw & early \\
\hline 60:1 [L 60:3] & סحش1 & סحشس & early \\
\hline 60:7 [L 60:9] & pio:0 & om waw & early \\
\hline 60:8 [L 60:10] & Doo & om initial waw & early \\
\hline 60:10 [L 60:12] & of & סحشس & early \\
\hline 61:6 [L 61:7] & 1900i & Nacoio & early \\
\hline 61:7 [L 61:8] & Nمص & ومهم & early \\
\hline 62:8 [L 62:9] & مע & pr bèth & early \\
\hline 63:9 [L 63:10] & مسحم, & om initial waw & early \\
\hline 64:4 [L 64:5] & 200 & om waw & early \\
\hline 65:5 [L 65:6] & حبم & حسس & early \\
\hline $66: 5$ & olg, & om dälath & early \\
\hline $66: 7$ & حمץ & om bèth & early \\
\hline $66: 8$ & isalo & Nano & early \\
\hline $66: 18$ & او:مسس & اوزمس & early \\
\hline 68:2 [L 68:3] & أָ & pr waw & early \\
\hline
\end{tabular}




\begin{tabular}{|c|c|c|c|}
\hline $\begin{array}{l}\text { Place } \\
68: 9 \text { [L 68:10] }\end{array}$ & $\begin{array}{l}\text { Mosul } \\
\text { aAch }\end{array}$ & $\begin{array}{l}\text { Leiden } \\
\text { oisal }\end{array}$ & $\begin{array}{l}\text { Ms support for } \mathbf{M} \\
\text { early }\end{array}$ \\
\hline 68:10 [L 68:11] & حمحتج & حمت:ت & early \\
\hline 68:16 [L 68:17] & $1 ; 0 \delta^{4}$ & pr bèth & early \\
\hline 68:18 [L 68:19] & منمحى & Nowo & early \\
\hline 68:21 [L 68:22] & تح| & $\begin{array}{l}\text { pr waw; plene } \\
\text { spelling Iحتى احت. }\end{array}$ & early \\
\hline 68:22 [L 68:23] & Tail & أوم & early \\
\hline 68:34 [L 68:35] & o خم::ط" & Wienio & early \\
\hline 69:6 [L 69:7] & $2 \mathrm{~N}$ & pr waw & early \\
\hline 69:17 [L 69:18] & No & om waw & late \\
\hline 69:21 [L 69:22] & ماصه حي. & pr bèth & early \\
\hline 69:27 [L 69:28] & هa & حa & 12a1 (مه) \\
\hline 69:28 [L 69:29] & of जo & om initial waw & early \\
\hline 70:5 [L 70:6] & من:ا & וגם & late \\
\hline $71: 2$ & محروسعابر & om initial waw & early \\
\hline $71: 6$ & הلحب & pr dālath & early \\
\hline $71: 10$ & إحה & pr waw & early \\
\hline $71: 15$ & موهمس & om initial waw & early \\
\hline $71: 16$ & Mari, & om dälath & early \\
\hline $71: 19$ & وبماب & مروبمعاب & early \\
\hline $71: 20$ & 9) & pr waw & early \\
\hline $71: 20$ & booal & ouم & early \\
\hline $71: 22$ & 9io & om waw & early \\
\hline $71: 24$ & حمس & pr waw & early \\
\hline $72: 9$ & لحسه, & لحصف & early \\
\hline $72: 11$ & נסצ- & pr waw & early \\
\hline $72: 16$ & له & pr waw & early \\
\hline $72: 16$ & م, & م,صد| & early \\
\hline $73: 6$ & iامهaه & iliolow & $12 \mathrm{a} 1^{3}$ \\
\hline $73: 14$ & حم:| & خو:| & early \\
\hline 73:27 [L 73:28] & Wio & om waw & late \\
\hline $74: 1$ & 6רش & סحشس & early \\
\hline $74: 3$ & حمع ملا & ححه مبر & early \\
\hline
\end{tabular}

3 Several manuscripts read the plural, although they contain the initial waw: $8 \mathrm{a}^{\mathrm{c}}$, 9a1, 9t1.3, 10t1-3, 12t3.7; cf. Lee. 


\begin{tabular}{|c|c|c|c|}
\hline $\begin{array}{l}\text { Place } \\
74: 8\end{array}$ & $\begin{array}{l}\text { Mosul } \\
\text { o: }>\text { io }\end{array}$ & $\begin{array}{l}\text { Leiden } \\
\text { 0; } 1\end{array}$ & $\begin{array}{l}\text { Ms support for } \mathbf{M} \\
\text { early }\end{array}$ \\
\hline $74: 9$ & لما0 & om waw & early \\
\hline $74: 12$ & 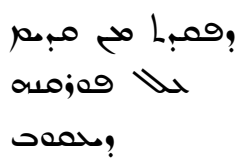 & 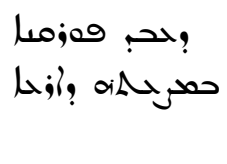 & early \\
\hline $74: 17$ & مامهتر & ماتمهسنه & early \\
\hline $74: 20$ & وت:هنه & وت:| & early \\
\hline 75:5 [L 75:6] & U & pr waw & early \\
\hline 75:6 [L 75:7] & لما0 & om waw & early \\
\hline 75:10 [L 75:11] & مذهم & محهاםم & early \\
\hline 76:4 [L 76:5] & סمحس & סممسس & early \\
\hline 77:1 [L 77:2] & مخد & pr bèth & early \\
\hline 77:5 [L 77:6] & ممحى & pr waw & early \\
\hline 77:5 [L 77:6] & مب.مر & مبرן & Lee, Urmia \\
\hline 77:8 [L 77:9] & allof & oflat. مس & early \\
\hline 77:8 [L77:9] & مסملهم & of of oملم & early \\
\hline $78: 3$ & 9io & om waw & early \\
\hline $78: 4$ & أمسنا & أمن:ما & early \\
\hline $78: 6$ & ! & pr lāmadh & early \\
\hline $78: 6$ & أمن:ا & أمن:ما & early \\
\hline $78: 17$ & $900 \%$ & O90010 & Urmia \\
\hline $78: 21$ & 9io & om waw & early \\
\hline 78:28 [L 78:29] & مأحه & om initial waw & early \\
\hline 78:38 [L 78:39] & مiolo & ألموح: & early \\
\hline 78:55 [L 78:56] & لمهם & pr waw & early \\
\hline 78:63 [L 78:64] & مهتصمן & pr waw & early \\
\hline 78:68 [L 78:69] & ? & istiod & early \\
\hline 78:69 [L 78:70] & 0>حما & om waw & early \\
\hline 78:70 [L 78:71] & ومستم & ومسما & early \\
\hline $79: 1$ & 0حصب00 & om initial waw & early \\
\hline $79: 4$ & م0عهما & om initial waw & early \\
\hline $79: 8$ & كمب:مهم & كمبمعسس & early \\
\hline $79: 9$ & حب9: & حبز & early \\
\hline $79: 9$ & معم & مهس & early \\
\hline $79: 9$ & م9ى & موى & early \\
\hline $79: 10$ & حسم & حستم & no support \\
\hline 80:11 [L 80:12] & קטب:ته & קטب:ه & no support \\
\hline
\end{tabular}




\begin{tabular}{|c|c|c|c|}
\hline $\begin{array}{l}\text { Place } \\
\text { 80:16 [L 80:17] }\end{array}$ & Mosul & $\begin{array}{l}\text { Leiden } \\
\text { pr waw }\end{array}$ & $\begin{array}{l}\text { Ms support for } \mathbf{M} \\
\text { early }\end{array}$ \\
\hline 80:18 [L 80:19] & U, & No & early \\
\hline 80:18 [L 80:19] & أمى & أمسس & early \\
\hline 80:18 [L 80:19] & No & N, & early \\
\hline 81:5 [L 81:6] & alogaco & Llo, 000 & early \\
\hline 81:13 [L 81:14] & a حصه & Ta & no support \\
\hline $82: 5$ & No & U, & early \\
\hline 83:15 [L 83:16] & حححتتب & حدخף & early \\
\hline 83:17 [L 83:18] & 0سف:0 & om initial waw & early \\
\hline 84:3 [L 84:4] & 9io & om waw & early \\
\hline 84:6 [L 84:7] & 9io & om waw & early \\
\hline 85:3 [L 85:4] & الححنا & pr waw & early \\
\hline 85:6 [L 85:7] & أوب & أوسس & early \\
\hline 85:6 [L 85:7] & 010م & oأسس & early \\
\hline 85:7 [L 85:8] & مa & مaw & early \\
\hline 85:10 [L 85:11] & أزهمן & ? زلحس & early \\
\hline 85:12 [L 85:13] & 9io & om waw & early \\
\hline 85:13 [L 85:14] & وبمل & pr waw & early \\
\hline $86: 5$ & wa & om & early \\
\hline $86: 8$ & וגם & ורם & early \\
\hline $86: 17$ & محص: & om waw & early \\
\hline $87: 6$ & حمده & ححقمما & early \\
\hline 88:14 [L 88:15] & anfle & الها & early \\
\hline 89:2 [L 89:3] & Li? & iol? & early \\
\hline 89:8 [L 89:9] & y & om initial waw & early \\
\hline 89:21 [L 89:22] & 9io & om waw & early \\
\hline 89:34 [L 89:35] & مبمر & pr waw & early \\
\hline 89:44 [L 89:45] & حهدي & pr waw & early \\
\hline 89:45 [L 89:46] & حفا & pr waw & early \\
\hline $90: 8$ & دلهم & حلمسس & early \\
\hline $90: 10$ & caly-000 & 20000 & early \\
\hline $90: 12$ & أموحي & أوبس & early \\
\hline $90: 14$ & محمي & محسس & early \\
\hline $91: 4$ & Lanlo & Sulo & Urmia \\
\hline 92:6 [L 92:7] & סקح: & om waw & early \\
\hline $93: 5$ & حهיקו & om lammadh & early \\
\hline $94: 8$ & ححما & وحمال & late \\
\hline
\end{tabular}




\begin{tabular}{|c|c|c|c|}
\hline $\begin{array}{l}\text { Place } \\
95: 9\end{array}$ & $\begin{array}{l}\text { Mosul } \\
\text { oro }\end{array}$ & $\begin{array}{l}\text { Leiden } \\
\text { om initial waw }\end{array}$ & $\begin{array}{l}\text { Ms support for } \mathbf{M} \\
\text { early }\end{array}$ \\
\hline $96: 5$ & من:ما & pr waw & early \\
\hline $96: 12$ & شهمع & لحقع & early \\
\hline $96: 12$ & r:00 & ea & early \\
\hline $97: 1$ & منتمع & om waw & late \\
\hline $97: 10$ & ححمه| & seyāme & early \\
\hline $98: 9$ & حمهa| & حرومعها| & early \\
\hline 102:18 [L 102:19] & أمن:ل & أمن:ما & early \\
\hline 102:18 [L 102:19] & محمار & om waw & early \\
\hline 102:19 [L 102:20] & حل أزحا & لإزحا & early \\
\hline 103:5 & مסسبا & om waw & early \\
\hline 103:12 & ?زمف & لمنم & early \\
\hline 103:16 & Ulo & om waw & early \\
\hline 104:1 & ה:حى & ה:ح & early \\
\hline 104:1 & ححمى & ححص & early \\
\hline 104:2 & Solio & i iمسم & early \\
\hline $104: 2$ & مهمد & مهم & early \\
\hline $104: 3$ & متهر & متونגه & early \\
\hline $104: 7$ & م) & om waw & early \\
\hline 104:10 & aم:نا & aم:; & early \\
\hline 104:12 & م & om waw & early \\
\hline 104:12 & ف:مش| & |0:01/| & early \\
\hline 104:13 & +o & om waw & early \\
\hline 104:16 & " & و & early \\
\hline 104:17 & مدنه & مده & early \\
\hline $104: 24$ & حiن & حصبـا & early \\
\hline $104: 25$ & La & sa & early \\
\hline 104:29 & مסحفو, & om waw & no support \\
\hline $105: 8$ & لكاوم: & مهوم: & early \\
\hline 105:11 & 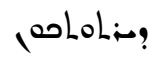 & poatoling & early \\
\hline 105:18 & أסـ:0 & : & early \\
\hline $105: 22$ & خمخها & مح>تها & early \\
\hline $105: 25$ & T000 & & early \\
\hline $105: 31$ & Lio & - Nio & early \\
\hline $105: 34$ & Nhio & Alio & early \\
\hline $105: 35$ & أمحه & '? Wh & Lee, Urmia \\
\hline 106:3 & ILeag! & aleog! & early \\
\hline
\end{tabular}




\begin{tabular}{|c|c|c|c|}
\hline $\begin{array}{l}\text { Place } \\
106: 4\end{array}$ & $\begin{array}{l}\text { Mosul } \\
\text { الووصنم }\end{array}$ & $\begin{array}{l}\text { Leiden } \\
\text { ألوحن:س }\end{array}$ & $\begin{array}{l}\text { Ms support for } \mathbf{M} \\
\text { early }\end{array}$ \\
\hline $106: 4$ & مه:00مى & مون:مسس & early \\
\hline 106:5 & وبسر| & 9ill & early \\
\hline 106:5 & مسب| & أمبا| & early \\
\hline $106: 5$ & 0سمححس & Aalo & early \\
\hline $106: 7$ & & 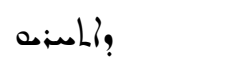 & early \\
\hline 106:9 & > & om waw & early \\
\hline $106: 24$ & حمده بمناً & حمحNa & no support \\
\hline $106: 26$ & pis & pr waw & early \\
\hline $106: 29$ & مانحم & محم & early \\
\hline $106: 31$ & مبمها & om waw & early \\
\hline $106: 37$ & موحسه & om initial waw & early \\
\hline 106:39 & agiflio & om initial waw & early \\
\hline 106:44 & حامختيتهم & حاه حيدهر & early \\
\hline $106: 45$ & alosof? & seyāme & early \\
\hline $106: 47$ & مصمى & סכטمس & early \\
\hline $107: 4$ & a) & pr waw & early \\
\hline $107: 9$ & مهقد| موتى| & مهوما موبد| & early \\
\hline $107: 12$ & oa:Dli & pr waw & early \\
\hline 107:22 & محسه & pr waw & early \\
\hline $107: 23$ & محصبت & סححتת & early \\
\hline $107: 23$ & حصب| & حص:| & early \\
\hline 107:29 & odao & olno & early \\
\hline 107:30 & موح: & om waw & late \\
\hline $107: 34$ & ه|ز" & هلزته & late \\
\hline $107: 38$ & וגה: & iا & early \\
\hline 108:6 [L 108:7] & ف:00: & ه:00:س & no support \\
\hline 108:6 [L 108:7] & محي & محس & no support \\
\hline 108:9 [L 108:10] & مoreاح & مهأح & early \\
\hline 109:2 & ? & iافيس & early \\
\hline 109:11 [L 109:12] & ومن:مف & ومن:مص & early \\
\hline 109:12 [L 109:13] & أمن:ل & أمن:ما & early \\
\hline 109:17 [L 109:18] & Who & حهد & early \\
\hline 109:21 [L 109:22] & ححس & prwaw & early \\
\hline 109:26 [L 109:27] & פוֹדיד & 9.9דף & early \\
\hline 111:7 [L 111:8a] & مسمى & pr waw & early \\
\hline 111:8 [L 111:8b] & ححה: & pr waw & early \\
\hline
\end{tabular}




\begin{tabular}{|c|c|c|c|}
\hline $\begin{array}{l}\text { Place } \\
112: 6\end{array}$ & $\begin{array}{l}\text { Mosul } \\
\text { حله }\end{array}$ & $\begin{array}{l}\text { Leiden } \\
\text { pr waw }\end{array}$ & $\begin{array}{l}\text { Ms support for } \mathbf{M} \\
\text { early }\end{array}$ \\
\hline $112: 10$ & Nar & pr waw & early \\
\hline $113: 3$ & مب:مها & om waw & early \\
\hline $114: 6$ & $1 \ddot{0} 0$ & $1 \ddot{0}$ & Urmia \\
\hline 114:15 [L 115:7] & حامب:ت00م & pr waw & early \\
\hline 114:25 [L 115:17] & Vlo & om waw & early \\
\hline 115:4 [L 116:4] & of & of & early \\
\hline 115:8 [L 116:8] & وقب & وفري & early \\
\hline 115:19 [116:19] & محبة| & om waw & early \\
\hline 118:3 [L 119:3] & No & om waw & early \\
\hline 118:4 [L 119:4] & o:fug & o:fug! & early \\
\hline 118:42 [L 119:42] & $W L$ & pr waw & early \\
\hline 118:170 [L 119:170] & في & 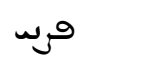 & no support \\
\hline 118:176 [L 119:176] & نمبا & حندا & early \\
\hline 118:176 [L 119:176] & أحبـLا & أحب| & early \\
\hline 119:1 [L 120:2] & مورينه & موفئه & early \\
\hline 120:6 [L 121:6] & لlo & om waw & early \\
\hline 121:4 [L 122:4] & مخق & ملهم & Lee, Urmia \\
\hline 122:2 [L 123:2] & 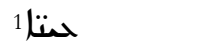 & حتس & early \\
\hline 122:2 [L 123:2] & و وحتبا & om dälath & early \\
\hline 122:2 [L123:2] & حتر2 & تسصنه & early \\
\hline 122:3 [L 123:3] & Lofha? & Lofla? & late \\
\hline 122:4 [L 123:4] & Lofuao & Laflas & early \\
\hline 123:3 [L 124:3] & مـ & pr waw & early \\
\hline 123:8 [L 124:8] & حهوزק & محهوزק & early \\
\hline 124:4 [L 125:4] & ملامح & om waw & early \\
\hline 124:5 [L 125:5] & مaحما & om waw & late \\
\hline 124:5 [L 125:5] & Whi Wa & لإمس:طل & early \\
\hline 126:1 [L 127:1 & 23 & pr waw & early \\
\hline 126:3 [L 127:3] & ومن:حار & وحم:حدا & early \\
\hline 127:6 [L 128:6] & ג " & لالمع:ط" & early \\
\hline 128:2 [L 129:2] & حسגد & om bèth & early \\
\hline 128:6 [L 129:6] & 100010 & 10000 & early \\
\hline 128:7 [L 129:7] & لlo & om waw & early \\
\hline 130:2 [L 131:2] & , & om dälath & early \\
\hline 131:4 [L 132:4] & Vlo & om waw & early \\
\hline 131:12 [L 132:12] & 9io & om waw & early \\
\hline
\end{tabular}




\begin{tabular}{|c|c|c|c|}
\hline $\begin{array}{l}\text { Place } \\
\text { 134:4 [L 135:4] }\end{array}$ & 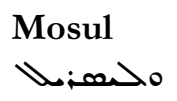 & $\begin{array}{l}\text { Leiden } \\
\text { Wienio }\end{array}$ & $\begin{array}{l}\text { Ms support for } \mathbf{M} \\
\text { early }\end{array}$ \\
\hline 134:12 [L 135:12] & vaso & om waw & early \\
\hline 135:15 [L 136:15] & - $9: 6$ & $9: 60$ & early \\
\hline 136:6 [L 137:6] & لبحم & 0ب:حم & early \\
\hline 138:16 [L 139:16] & oll & om waw & early \\
\hline 138:22 [L 139:22] & مoرام الم & مسلLال & early \\
\hline 139:3 [L 140:4] & Lont & Lus & Urmia \\
\hline 139:3 [L 140:4] & مهقام0aر & حمد00, & early \\
\hline 139:11 [L 140:12] & סקح: & om waw & early \\
\hline 142:5 [L 143:5] & ; & pr waw & early \\
\hline 142:12 [L 143:12] & حهحهاب & محهحماب & early \\
\hline 143:8 [L 144:8] & סעل & ممحلط" & early \\
\hline 143:10 [L 144:10] & Now? & ! & early \\
\hline 143:11 [L 144:11] & סעل & ممخلص & early \\
\hline 143:12 [L 144:12] & حته0 & حتر & early \\
\hline 143:12 [L 144:12] & مخصه & محتّ & early \\
\hline 143:13 [L 144:13] & oörol & ماةم & early \\
\hline 143:13 [L 144:13] & حتهمر & حم & early \\
\hline 143:13 [L 144:13] & حمعمتهر & حقهمب & early \\
\hline 143:14 [L 144:14] & حمت:001 & حمتץ & early \\
\hline 143:14 [L 144:14] & وحم:ا & وحمتر & early \\
\hline 143:14 [L 144:14] & llo & om waw & Urmia \\
\hline 143:14 [L 144:14] & حمعمتِ00م & حقهمي & early \\
\hline 143:15 [L 144:15] & floag? & pag & Urmia \\
\hline 144:10 [L 145:11] & مaعحس & om waw & early \\
\hline 144:15 [L 145:15] & ! ?ars & om dälath & early \\
\hline 145:5 [L 146:5] & إلحا & 10011 & early \\
\hline 145:7 [L 146:7] & حح: & pr waw & early \\
\hline 146:8 [L 147:8] & مaso & om waw & early \\
\hline 146:8 [L 147:8] & معدا & pr waw & early \\
\hline 146:9 [L 147:9] & sas & pr waw & early \\
\hline 147:13 & وأحم & وحمده & early \\
\hline $147: 14$ & oمص & om waw & early \\
\hline $147: 16$ & مخ:هلا & io> דה:1 & early \\
\hline $147: 20$ & lea & 2a & Lee, Urmia \\
\hline 148:6 & مامص & om waw & early \\
\hline $148: 13$ & oncحسه & om initial waw & early \\
\hline
\end{tabular}




\begin{tabular}{|c|c|c|c|}
\hline $\begin{array}{l}\text { Place } \\
149: 4\end{array}$ & $\begin{array}{l}\text { Mosul } \\
\text { 9هزمسا }\end{array}$ & $\begin{array}{l}\text { Leiden } \\
\text { هم:مسه }\end{array}$ & $\begin{array}{l}\text { Ms support for } \mathbf{M} \\
\text { early }\end{array}$ \\
\hline 149:6 & مـنمخ;مه & لـمحزمه & early \\
\hline $150: 4$ & محتحدا & om waw & Lee, Urmia \\
\hline $150: 6$ & حصل & om bèth & early \\
\hline $150: 6$ & لمحس & مامحس & early \\
\hline
\end{tabular}

